\title{
Costo de la atención materno infantil en el estado de Mor elos, México
}

\author{
Lucero C ahuana-H urtado, M en C, ${ }^{(1)}$ Sandra Sosa-Rubí, M en C, ${ }^{(1)}$ \\ Stefano Bertozzi, MD, PhD.(1)
}

\section{Cahuana-Hurtado L, Sosa-Rubí S, Bertozzi S. Costo de la atención materno infantil en el estado de Morelos, México.}

Salud Publica Mex 2004:46:316-325. El texto completo en inglés de este artículo está disponible en: http://www.insp.mx/salud/index.html

\section{Resumen}

Objetivo. Comparar el costo actual de servicios de salud materno infantiles (modelo actual) con el costo hipotético de su provisión bajo estándares definidos por el Paquete Madre Bebé, de la 0 rganización Mundial de la Salud. Material y métodos Se realizó un estudio piloto de caso, de corte transversal, en septiembre de 2001, en establecimientos de salud de la Jurisdicción Sanitaria III de los Servicios de Salud del Estado de Morelos, México. Se eligieron para el estudio dos centros de salud rurales, un centro de salud urbano y un hospital general de la Secretaría de Salud. Se calculó el costo total y por intervención del modelo actual y del modelo Paquete Madre Bebé, usando el Modelo de estimación de costos de este Paquete. Resultados Se encontró que el costo total del Paquete Madre Bebé es dos veces mayor que el costo del modelo actual. D e las 18 intervenciones evaluadas, la atención prenatal y el parto no rmal consumen la mayor proporción de los costos totales. Los costos de personal representan más de la mitad de los costos totales. Conclusiones. El Modelo de estimación de costos es una her ramienta práctica para la estimación y comparación de costos, y es útil para guiar la distribución de recursos financieros destinados a la atención materno infantil. Sin embargo, su aplicación es de limitada utilidad si su adaptación no responde a las características de cada sistema de atención. El texto completo en inglés de este artículo está disponible en: http://www.insp.mx/salud/index.html

Palabras clave: costos de la atención en salud; salud materno infantil; embarazo; parto; México

\author{
Cahuana-Hurtado L, Sosa-Rubí S, Bertozzi S. \\ Cost of mother-child care \\ in Morelos State. \\ Salud Publica Mex 2004;46:316-325. \\ The English version of this paper \\ is available at: http://www.insp.mx/salud/index.html
}

\begin{abstract}
Objective.To compare the cost of maternal and child health care (current model) to that of the W HO Mother-Baby Package if it were implemented. Material and Methods A pilot cross-sectional case study was conducted in September 2001 in Sanitary D istrict N o. III, Morelos State, Mexico. Two rural health centers, an urban health center, and a general hospital, all managed by the Ministry of Health, were selected for the study. The Mother-Baby Package Costing Spreadsheet was used to estimate the total cost and cost per intervention for the current model and for the M otherBaby Package model. Results. The total cost of the Mother-Baby Package was twice the cost of the current model. of the 18 interventions evaluated, the highest proportion of total costs corresponded to antenatal care and normal delivery. Personnel costs represented more than half of the total costs. Conclusions The M other-Baby Package Costing Spreadsheet is a practical tool to estimate and compare costs and is useful to guide the distribution of financial resources allocated to maternal and child healthcare. However, this model has limited application unless it is adapted to the structure of each healthcare system.The English version of this paper is available at:http://www.insp.mx/salud/index.html
\end{abstract}

Key words: health care costs; maternal and child health; pregnancy; delivery; Mexico

(1) Instituto N acional de Salud Pública, Centro de Investigación en Sistemas de Salud, D irección de Economía y Políticas de la Salud. Cuernavaca, Morelos, México.

Fecha de recibido: 2 de octubre de 2003 - Fecha de aprobado: 8 de junio de 2004

Solicitud de sobretiros: Lucero Cahuana Hurtado. Instituto N acional de Salud Pública. Centro de Investigación en Sistemas de Salud. Avenida Universidad 655 colonia Santa María A huacatitlán 62508. Cuernavaca, Morelos, México.

Correo electrónico: Icahuana@ insp.mx. 
E n el mundo, cada año mueren más de 550 mil mujeres por complicaciones durante el embarazo o el parto. ${ }^{1}$ Una importante proporción de estas muertes se debe a la falta de atención médica otorgada por personal capacitado. ${ }^{1}$ En México, como en otros países en desarrollo, cada día cuatro mujeres en edad reproductiva mueren por causas obstétricas, ${ }^{2}$ a pesar de los esfuerzos realizados para mejorar el acceso a los servicios de salud y la calidad de la atención materno infantil. ${ }^{3}$ Este problema se concentra en zonas rurales donde el riesgo de morir es tres veces mayor que en zonas urbanas. ${ }^{4}$

Ante esta problemática, y siguiendo las recomendaciones para atender el parto con personal calificado, ${ }^{5,6}$ la Organización Mundial de la Salud (OMS) y el Fondo de las Naciones Unidas para la Infancia (UNI$\mathrm{CEF})^{7}$ desarrollaron en 1994 un paquete de intervenciones básicas en salud para reducir la mortalidad y morbilidad materna y neonatal, llamado Paquete Madre Bebé (PMB). En dicho paquete se sugieren 18 intervenciones que han probado ser costo-efectivas en la reducción de muertes maternas. ${ }^{7}$ Estos estándares representan una combinación esencial de insumos que, según la experiencia africana, aseguran un nivel de calidad básica, asumiendo la existencia de un sistema de referencia y contrarreferencia funcional. ${ }^{7}$ Incluye el libro en Microsoft Excel ${ }^{\circledR}, 8$ Modelo de estimación de $\operatorname{costos}^{9}$ el cual, basado en parámetros de regiones de bajos recursos, recrea los componentes del costo financiero de la provisión de servicios materno infantiles en un sistema de tres niveles de atención de salud. Al comparar dos situaciones alternativas (la situación actual y una hipotética en la que se brinda atención bajo los protocolos del PMB) permite calcular la inversión necesaria para proveer servicios materno infantiles de acuerdo con el nivel recomendado por el Paquete. ${ }^{10}$

El Modelo de estimación de costos ha sido aplicado en Bolivia ${ }^{11}$ y Uganda ${ }^{12}$ para analizar la aplicación de las recomendaciones del PMB. Los resultados de dichos estudios permitieron obtener información que fue útil para solicitar mayores recursos y buscar nuevas fuentes de financiamiento para los programas nacionales de salud materno infantil de estos países. En el caso mexicano, el único esfuerzo ${ }^{13}$ de utilizar esta metodología estriba en un estudio piloto en un centro de salud rural en el estado de Morelos, en el cual se dejaron de lado otros niveles de atención.

Debido al interés del gobierno mexicano en aplicar políticas similares a las recomendadas por el PMB en estrategias prioritarias como el programa de Arranque Parejo en la Vida ${ }^{14}$ y el Seguro Popular de Salud, ${ }^{15}$ y a la importancia de conocer el costo de la atención materno infantil en diferentes niveles de atención en México, el presente trabajo busca estimar el costo financiero o gasto de la provisión de atención materno infantil, usando el Modelo de estimación de costos del PMB. Además, se compara el costo actual de la atención materno infantil con el costo hipotético de la aplicación del PMB en México. Para ello se amplía el número de unidades de salud estudiadas, con el objetivo de analizar la utilidad de dicho instrumento para la toma de decisiones económicas en la atención materno infantil.

\section{Material y métodos}

Se realizó un estudio piloto de caso, de corte transversal, en septiembre de 2001, en establecimientos de salud dentro de la zona de influencia de la Jurisdicción Sanitaria III de los Servicios de Salud del Estado de Morelos, México, con el objetivo de comparar el costo de la atención materno infantil actual al costo hipotético de la atención según los estándares del PMB. Se usó información sobre las características de los establecimientos de salud, insumos utilizados (medicinas, consumibles, horas de trabajo, laboratorio) para la atención de un paciente promedio por tipo de intervención en cada establecimiento estudiado, y los precios de éstos.

Se seleccionó una muestra a conveniencia, y se recolectó información en establecimientos de los Servicios de Salud del Estado de Morelos, adscritos a la Secretaría de Salud (SSA) ubicados en cuatro cabeceras municipales. Así, se eligieron dos centros de salud rurales (CSR), un centro de salud urbano (CSU) y un hospital (HG) con el fin de resaltar las diferencias en la utilización de recursos para la atención materno infantil en áreas rurales y urbanas, y en distintos niveles de atención de la salud.

Se consideraron centros de salud ubicados en municipios con alto nivel de marginación, según el XII Censo General de Población y Vivienda de 2000, ${ }^{16}$ y en los que la tasa de mortalidad materna fue superior a la estatal. ${ }^{*}$ Del mismo modo, se eligieron unidades de salud de primer nivel de atención localizadas a más de 30 minutos de distancia del hospital de referencia más cercano, ${ }^{\ddagger}$ pues se considera que a esa distancia deben existir servicios con capacidad de brindar aten-

* Servicios de Salud de Morelos. Oficina de Estadística. Entrevista. Cuernavaca, Morelos, México: SSM; 2001 septiembre 10.

¥ Servicios de Salud de Morelos. Estudio de regionalización operativa 2001. Cuautla, Morelos, México: Jurisdicción Sanitaria No. III, 2001. Documento interno de trabajo. 
ción básica para emergencias obstétricas. ${ }^{17}$ Por otro lado, se eligió el hospital al cual los centros de salud seleccionados regularmente refieren a los pacientes.

Para llevar a cabo esta investigación se obtuvo autorización para el levantamiento de información de los Servicios de Salud del Estado de Morelos y la aprobación de la Comisión de Investigación, del Instituto Nacional de Salud Pública. Posteriormente, se solicitó el apoyo de la Jurisdicción Sanitaria No III para la recolección de información en cada establecimiento de salud.

\section{El Modelo de estimación de costos del PMB}

El Modelo de estimación de costos del $\mathrm{PMB}^{9}$ es un libro de Microsoft Excel ${ }^{\circledR},{ }^{8}$ cuyas hojas se encuentran interrelacionadas para estimar los costos financieros o gastos proyectados de la provisión de 18 intervenciones de salud materno infantil en una región rural de bajos recursos.

Empleando información local, este modelo permite estimar el costo de los servicios actuales, y proyectar los costos por proveer servicios de salud materno infantiles de acuerdo con los estándares del PMB. La diferencia entre ambos costos representa la inversión adicional necesaria para fortalecer la provisión de servicios de salud.

El Modelo de estimación de costos distingue dos grandes categorías de costos: costos de capital, que se realizan una sola vez y no son variables (como la construcción de edificios o la compra de equipo grande), y costos variables o costos operativos. Los costos variables se subdividen en costos directos e indirectos. Los costos directos son aquellos que se pueden atribuir directamente a una intervención individual como medicinas, consumibles médicos y de laboratorio, días cama, productos sanguíneos, transporte y el tiempo que el personal médico dedica por paciente. Los costos indirectos son costos variables que no pueden ser atribuidos directamente a una intervención médica específica, como el costo de mantenimiento y uso de los edificios, los salarios del personal de apoyo, costos del mercadeo social y material educativo y de publicidad para el programa, así como el costo de supervisión. ${ }^{10}$

Se incluyen, para la región estudiada, estimaciones de costos totales, costos promedio per cápita y costos promedio por parto. Las estimaciones de costos pueden ser desagregadas por insumo (como medicinas, vacunas, salarios e infraestructura), por intervención (como el manejo de parto normal, hemorragia, eclampsia y sepsis), diferentes tipos de costos (costos variables y de capital), por nivel de establecimiento (hospital, centro de salud o puesto de salud), per cápita y por parto. ${ }^{10}$

El Modelo de estimación de costos se acompaña de una serie de cuestionarios para recolectar información, lo que permite capturar la forma como se lleva a cabo cada intervención (formato de recolección de datos del PMB). Asimismo, ante la necesidad de generar costos promedios a partir de datos para varios establecimientos de salud en un mismo nivel de atención, se incluye el documento Formato de consolidación de datos del PMB. Finalmente, se adjuntan archivos con resultados gráficos para facilitar el análisis de datos.

Se tradujeron al español todos los instrumentos incluidos en el Modelo de estimación de costos (anteriormente validados en el trabajo realizado para México), ${ }_{1}^{13}$ con el fin de aplicarlos al contexto mexicano. Adicionalmente, debido a que el sistema de atención local difiere del modelo PMB, se adaptó el Modelo de estimación de costos del PMB a las características del sistema de atención a población abierta, con el objetivo de hacer comparables los modelos PMB y actual.

En primer lugar, ya que el tipo de establecimientos en el modelo PMB (puesto de salud, centro de salud y hospital) no coincide con las características del grupo de unidades de salud estudiados (centro de salud rural, centro de salud urbano y hospital), se modificó el tipo de unidades de salud incluidas en el PMB. Así, en el espacio del "Puesto de salud" se tomó en cuenta el CSR. En segundo lugar, se consideró como "Centro de salud" la unidad de salud urbana estudiada, puesto que en ella se brindaban más servicios que en el CSR. Por último, se usó el espacio de "Hospital" para reflejar la situación del HG, por ser de mayor infraestructura que los CSR y los CSU. Todos estos cambios implicaron modificaciones en la infraestructura, actividades, personal, insumos y porcentajes de pacientes referidos, considerados en cada una de las intervenciones en cada tipo de establecimiento.

La recolección de datos se realizó en septiembre de 2001. En este periodo se visitaron los establecimientos de salud y se aplicaron cuestionarios (incluidos en el formato de recolección de datos del PMB) al personal de salud encargado de la atención materno infantil, y se preguntó por el uso de insumos por tipo de intervención (medicinas, consumibles, horas de trabajo, laboratorio) para la atención de un paciente promedio.

El proceso y la captura de datos se realizaron en octubre de 2001. Los datos para los CSR se transformaron utilizando el Formato de consolidación de datos del PMB para obtener los costos de un CSR promedio. Los costos para el CSU y el HG fueron considerados 
como costos promedio para la región estudiada. La información consolidada fue capturada en el Modelo de estimación de costos en Microsoft Excel, versión 2000. ${ }^{8}$

La información demográfica, de cobertura, epidemiológica y de infraestructura fue provista por los Servicios de Salud del Estado de Morelos, ${ }^{*, \neq, 18}$ por publicaciones de la SSA ${ }^{2}$ y cifras propuestas por la OMS.

Se usaron estimaciones de población de finales de 1999. Con base en datos de la SSA, se asumió que en el modelo actual $90 \%$ de todas las mujeres embarazadas recibía atención prenatal básica, $88 \%$ de todos los partos era atendido por personal calificado, ${ }^{14}$ además, se supuso una tasa de prevalencia de anticonceptivos de $62.1 \% .{ }^{19}$ En el caso del modelo estándar, se ingresaron las metas de cobertura del Programa Materno Infantil Mexicano 2001-2006. ${ }^{14}$

Los medicamentos y los insumos médicos fueron valorados de acuerdo con la lista de precios de la SSA en $1999 .{ }^{20}$ Para aquellos insumos de los que no se tuvo información acerca de su valor se tomaron como referencia los precios notificados por UNICEF. Los salarios del personal médico y de apoyo fueron obtenidos de la lista de salarios del Hospital General de Cuautla. Los beneficios adicionales, como el alojamiento y la alimentación, también se incluyeron.

Los precios de los medicamentos, insumos médicos, capital, edificios y salarios reportados para antes del año 2001 fueron deflactados de acuerdo con el comportamiento de sus índices respectivos. ${ }^{21}$ Para la conversión en dólares se consideró el tipo de cambio vigente a fines de 2001, de 9.30 pesos por dólar. ${ }^{22}$

Ante la imposibilidad de hacer un estudio de tiempos, se supuso que aproximadamente $30 \%$ del tiempo de trabajo del personal médico es tiempo muerto, de acuerdo a un estudio anterior realizado en la misma zona. ${ }^{13}$ Este supuesto permitió asumir que una enfermera o médico trabaja cinco de las ocho horas de la jornada laboral diaria. Los salarios mensuales ofrecidos por la SSA fueron divididos por el número de días laborables en el mes y el número de horas por día para obtener el costo por hora de cada personal.

Con el fin de determinar los insumos disponibles en los establecimientos de esta entidad, y hacer comparables los protocolos definidos por el PMB a la si-

\footnotetext{
* Servicios de Salud de Morelos. Oficina de Estadística. Entrevista. Cuernavaca, Morelos, México: SSM; 2001 septiembre 10.

¥ Servicios de Salud de Morelos. Estudio de regionalización operativa 2001. Cuautla, Morelos, México: Jurisdicción Sanitaria No. III, 2001. Documento interno de trabajo.
}

tuación actual, se revisó el listado de medicamentos y equipos básicos de la SSA.

Para el análisis de los datos se hizo un doble ejercicio de estimación de costos y se construyeron dos modelos de costos a comparar. El modelo actual se definió como el uso de recursos emprendido en el momento del estudio, en términos de sueldos y salarios, infraestructura, transporte de emergencia, costos de medicinas, insumos y consumibles. Por otra parte, el modelo PMB se definió como la atención materno infantil que resultaría luego de introducir las líneas de tratamiento recomendadas por la OMS en todas las intervenciones cubiertas por el PMB, de acuerdo con la población de estudio y asumiendo niveles de infraestructura, transporte, personal técnico y personal clínico capaces de resolver complicaciones obstétricas y neonatales.

Los resultados en costos de ambos modelos se compararon mediante gráficos y cuadros, empleando los archivos incluidos en el Modelo de estimación de costos. De esta manera, se generaron cuadros para el costo de cada intervención por insumo; el costo en cada tipo de establecimiento por insumo, por intervención, costo directo, indirecto y de capital, y el costo del PMB total, por parto y per cápita.

\section{Resultados}

Se estudiaron dos centros de salud rurales con dos núcleos básicos (es decir, dos enfermeras y dos médi$\cos )$, un centro de salud urbano de 10 núcleos básicos y un hospital general, que brinda atención a una población de 440000 habitantes.

Las intervenciones del PMB brindadas en cada establecimiento, según cada modelo, se encuentran detalladas en el cuadro I. No se incluye la inserción de Norplant ${ }^{\circledR}$ como método anticonceptivo (como lo supone el PMB) pues éste no se brinda en los establecimientos estudiados. Se observa que bajo el modelo PMB se propone una oferta de intervenciones adicionales a las ofrecidas actualmente en los CSR y CSU, como la esterilización femenina, en el CSR, y la atención de complicaciones de aborto y sepsis, en el CSU. Por otro lado, se sugiere que el HG brinde todas las intervenciones del $\mathrm{PMB}$, a diferencia de la situación actual, en la que las pacientes que se acercan al hospital a solicitar las intervenciones más simples son referidas al centro de salud más cercano a su domicilio, (cuadro I).

Respecto al costo total, los resultados de este estudio muestran que con la aplicación hipotética del modelo PMB, comparado con el costo del modelo actual, el costo de la atención materno infantil aumenta- 
ría hasta dos veces. Esta situación se presenta en todos los componentes del costo (costo directo, indirecto y de capital) así como en todos los tipos de establecimientos (cuadro II).

Existe un grupo de intervenciones en las cuales hay diferencias en el costo total en el modelo actual y el modelo PMB. En el CSR se observa que la atención del parto normal, la atención prenatal, el tratamiento de las infecciones de transmisión sexual (ITS) distintas a la sífilis, la inserción del dispositivo intrauterino (DIU) y la provisión de condones son más costosas bajo el modelo PMB, mientras que la atención posparto es menos costosa (cuadro III). En el CSU, los costos del modelo PMB son más altos para la inserción del DIU, la atención del parto normal, la provisión de condones y el tratamiento de la sífilis. Por último, en el HG resalta el aumento en el costo de métodos de planificación familiar, como la inserción del DIU, la provisión de condones y de Depoprovera ${ }^{\circledR}$, así como la disminución de la utilización de los recursos para la atención de partos normales cuando el modelo PMB es aplicado (cuadro III).

Muchos de los cambios mencionados se deben a una redistribución de los pacientes entre los diferentes establecimientos de salud (cuadro III), como también a variaciones en el costo directo por caso (cuadro IV).

Cuando se analizan los costos por tipo de insumo, se observa que la aplicación del modelo PMB propone una inversión adicional para la contratación de personal médico, paramédico, y para la compra de medicamentos en todos los establecimientos analizados (cuadro V).
Cuadro I

INTERVENCIONES BRINDADAS, SEGÚN EL MODELO ACTUAL

E INTERVENCIONES A SER BRINDADAS POR EL MODELO

del Paquete Madre Bebé, por tipo

DE eStABlecimiento, JuRISDicción SANitARIa III. Morelos, México, 2001

\section{Centro de Centro de Hospita salud rural salud urbano general Actual PMB Actual PMB Actual PMB}

\begin{tabular}{lcccccc}
\hline Anemia severa & & & & & $X$ & $X$ \\
\hline Atención de parto normal & & $X$ & $X$ & $X$ & $X$ & $X$ \\
\hline Atención posparto & $X$ & $X$ & $X$ & $X$ & & $X$ \\
\hline Atención prenatal & $X$ & $X$ & $X$ & $X$ & & $X$ \\
\hline Complicaciones de aborto & & & & $X$ & & $X$ \\
\hline Complicaciones neonatales & & & & & $X$ & $X$ \\
\hline Eclampsia & & & & & $X$ & $X$ \\
\hline ITS-sífilis & $X$ & $X$ & $X$ & $X$ & & $X$ \\
\hline ITS-otras & $X$ & $X$ & $X$ & $X$ & & $X$ \\
\hline Hemorragia & & & & $X$ & $X$ & $X$ \\
\hline Parto prolongado/ obstruido & & & & & $X$ & $X$ \\
\hline PF-anticonceptivos orales & $X$ & $X$ & $X$ & $X$ & & $X$ \\
\hline PF-condón & $X$ & $X$ & $X$ & $X$ & & $X$ \\
\hline PF-depoprovera & $X$ & $X$ & $X$ & $X$ & & $X$ \\
\hline PF-DIU & $X$ & $X$ & $X$ & $X$ & $X$ & $X$ \\
\hline PF-esterilización & & & & & $X$ & $X$ \\
\hline Sepsis & & & & $X$ & $X$ & $X$ \\
\hline Total de intervenciones & 8 & 9 & 9 & 12 & 9 & 17
\end{tabular}

Notas:

ITS: infecciones de transmisión sexual

PF: planificación familiar

DIU: dispositivo intrauterino

Cuadro II

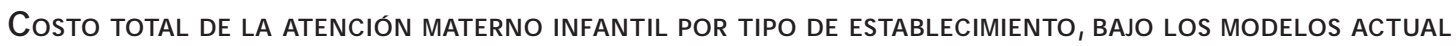
y del Paquete Madre Bebé, según componente de costos, Jurisdicción Sanitaria III. Morelos, México, 2001

\begin{tabular}{|c|c|c|c|c|c|c|c|c|}
\hline & \multicolumn{2}{|c|}{ Centro de salud rural } & \multicolumn{2}{|c|}{ Centro de salud urbano } & \multicolumn{2}{|c|}{ Hospital general } & \multicolumn{2}{|c|}{ Total } \\
\hline & $\begin{array}{c}\text { Actual } \\
\text { Est=40 } \\
\mathrm{Pac}=12425\end{array}$ & $\begin{array}{c}\text { PM B } \\
E s t=50 \\
P a c=18245\end{array}$ & $\begin{array}{c}\text { Actual } \\
\text { Est }=15 \\
\mathrm{Pac}=8288\end{array}$ & $\begin{array}{c}\mathrm{PMB} B \\
\mathrm{Est}=20 \\
\mathrm{Pac}=12486\end{array}$ & $\begin{array}{c}\text { Actual } \\
\text { Est }=2 \\
\mathrm{Pac}=6641\end{array}$ & $\begin{array}{c}\mathrm{PMB} \\
\mathrm{Est}=2 \\
\mathrm{Pac}=7578\end{array}$ & $\begin{array}{c}\text { Actual } \\
\text { Est }=47 \\
\operatorname{Pac}=27354\end{array}$ & $\begin{array}{c}\text { PMB } \\
E s t=72 \\
P a c=38309\end{array}$ \\
\hline Costo directo & $\$ 296.03$ & $\$ 653.98$ & $\$ 253.80$ & $\$ 399.74$ & $\$ 661.65$ & $\$ 796.63$ & $\$ 1211.48$ & $\$ 1850.35$ \\
\hline Costo indirecto & $\$ 88.47$ & $\$ 141.22$ & $\$ 234.05$ & $\$ 528.12$ & $\$ 90.65$ & $\$ 180.40$ & $\$ 413.17$ & $\$ 849.74$ \\
\hline Costo capital & $\$ 67.60$ & $\$ 110.16$ & $\$ 66.48$ & $\$ 152.14$ & $\$ 30.89$ & $\$ 56.31$ & $\$ 164.97$ & $\$ 318.61$ \\
\hline Costo total & $\$ 452.11$ & $\$ 905.36$ & $\$ 554.32$ & $\$ 1079.99$ & $\$ 783.19$ & $\$ 1033.34$ & $\$ 1789.61$ & $\$ 3018.69$ \\
\hline
\end{tabular}

Notas:

PMB: Paquete Madre Bebé

Est: número de establecimientos

Pac: número de pacientes

Costo en miles de dólares estadounidenses ( $1 \mathrm{USD}=9.30$ pesos mexicanos) 


\section{Cuadro III \\ Número de pacientes y costo total de los modelos actual y del Paquete Madre Bebé, por intervención en Cada tipo de establecimiento, JuRisdicción Sanitaria III. Morelos, México, 2001}

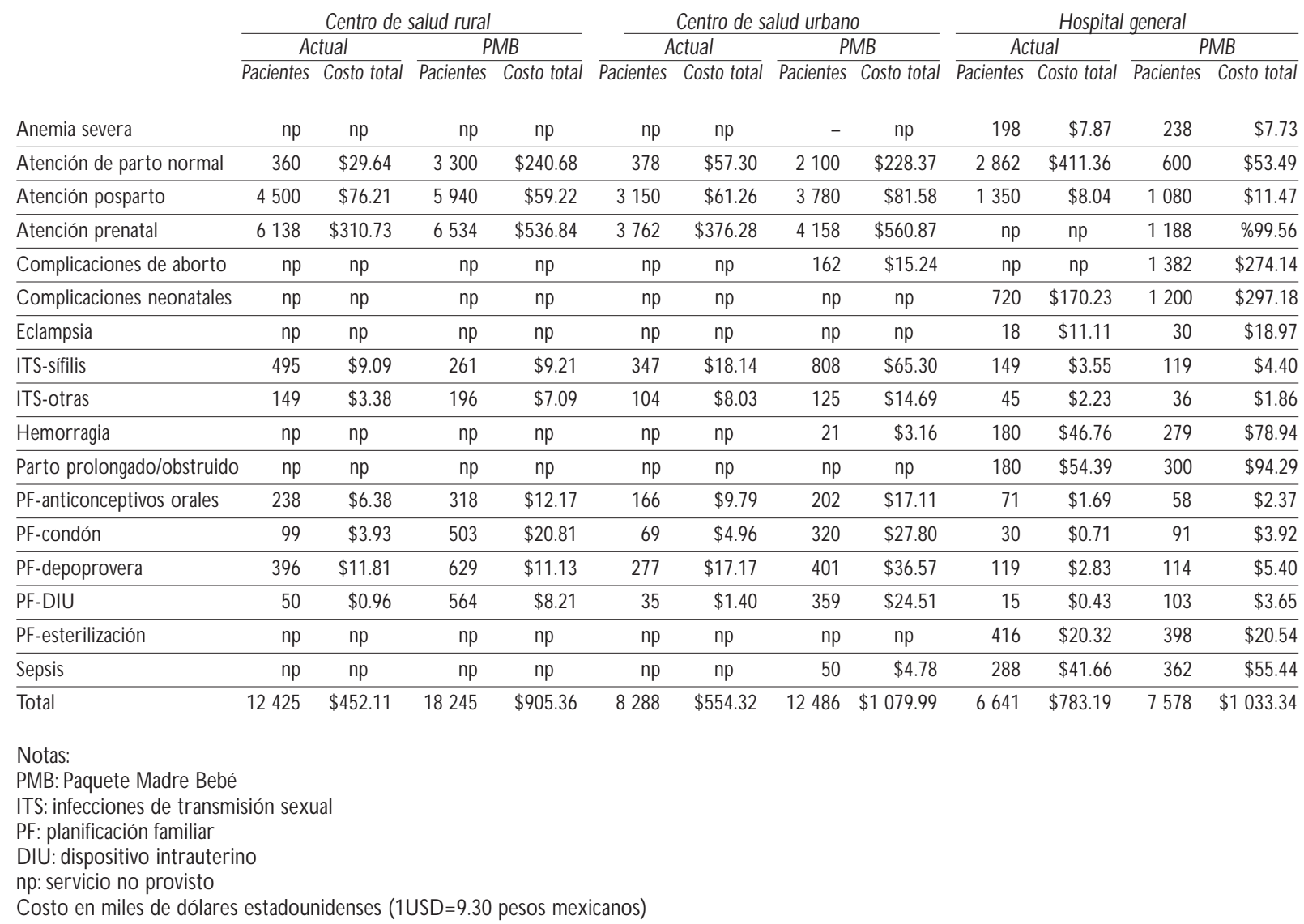

\section{Discusión}

En este estudio se encuentra que el costo hipotético de la atención materno infantil, bajo los protocolos de atención del (modelo PMB) es dos veces más alto que el costo total de los servicios materno infantiles provisto bajo la práctica actual en los establecimiento estudiados (modelo actual), en todos los componentes de costos y en todos los tipos de establecimientos examinados. Estos costos son inferiores a los encontrados en Bolivia ${ }^{11}$ y Uganda, ${ }^{12}$ donde debido a la necesidad de invertir en mayor equipo, infraestructura y capital humano y el nivel de pobreza existente en dichos países el costo del modelo PMB es 2.4 y 3 veces más alto, respectivamente, que el costo del modelo actual.

La diferencia entre el costo total en el modelo PMB y el costo actual se debe a múltiples factores. Uno de ellos es el incremento de la cobertura sugerido por el modelo PMB, que genera un aumento del total de pa- cientes atendidos y, por ende, del costo total, como lo menciona Sosa. ${ }^{13}$ La variación del costo total por intervención en cada tipo de establecimiento se debe, además, a la redistribución de pacientes propuesta por el $\mathrm{PMB}$, que indica que la atención de complicaciones se debe concentrar en el hospital, como se sugiere en otros estudios, asumiendo la existencia de un sistema de referencia funcional. ${ }^{23}$

De igual modo, respecto al modelo actual existe un aumento en el número de intervenciones prestadas y los servicios que las componen bajo el modelo PMB. Estos cambios se reflejan en variaciones en el costo directo por caso entre ambos modelos y son el parto normal, la atención prenatal, las hemorragias y las complicaciones neonatales las intervenciones con mayor peso en el costo total, resultado similar al encontrado en estudios anteriores. ${ }^{11,12}$ Cabe mencionar que el costo directo por caso calculado para el modelo actual es similar al costo hallado en estudios realizados en Mé- 


\section{Cuadro IV \\ Costo diRecto Por CASO de LAS INTERVENCIONES BRINDADAS EN CADA ESTABLECIMIENTO, BAJO LOS MOdeLOS ACTUAL y del Paquete Madre Bebé, Jurisdicción Sanitaria III. Morelos, México, 2001}

\begin{tabular}{|c|c|c|c|c|c|c|}
\hline & Centr & & Centr & urbano & & \\
\hline & Actual & $\mathrm{PMB}$ & Actual & PMB & Actual & PMB \\
\hline Anemia severa & np & np & $\mathrm{np}$ & $\$ 8.48$ & $\$ 21.86$ & $\$ 11.66$ \\
\hline Atención de parto normal & $\$ 69.76$ & $\$ 72.93$ & $\$ 129.22$ & $\$ 72.93$ & $\$ 131.82$ & $\$ 75.27$ \\
\hline Atención posparto & $\$ 4.37$ & $\$ 3.21$ & $\$ 8.27$ & $\$ 3.67$ & np & $\$ 3.67$ \\
\hline Atención prenatal & $\$ 38.06$ & $\$ 55.12$ & $\$ 44.12$ & $\$ 45.35$ & $\mathrm{np}$ & $\$ 42.15$ \\
\hline Complicaciones de aborto & np & $\mathrm{np}$ & $\mathrm{np}$ & $\$ 58.28$ & $\mathrm{np}$ & $\$ 170.59$ \\
\hline Complicaciones neonatales & np & $\mathrm{np}$ & $\mathrm{np}$ & $\mathrm{np}$ & $\$ 194.74$ & $\$ 199.05$ \\
\hline Eclampsia & $\mathrm{np}$ & $\mathrm{np}$ & $\mathrm{np}$ & $\mathrm{np}$ & $\$ 575.65$ & $\$ 583.82$ \\
\hline ITS-sífilis & $\$ 10.09$ & $\$ 9.16$ & $\$ 10.09$ & $\$ 10.09$ & $\$ 13.89$ & $\$ 10.09$ \\
\hline ITS-otras & $\$ 5.81$ & $\$ 8.26$ & $\$ 7.56$ & $\$ 9.19$ & np & $\$ 9.19$ \\
\hline Hemorragia & $\mathrm{np}$ & np & $\mathrm{np}$ & $\$ 114.57$ & $\$ 218.09$ & $\$ 234.33$ \\
\hline Parto prolongado/ obstruido & np & np & np & $\mathrm{np}$ & $\$ 260.51$ & $\$ 265.70$ \\
\hline PF-anticonceptivos orales & $\$ 14.23$ & $\$ 11.22$ & $\$ 14.23$ & $\$ 13.08$ & $\mathrm{np}$ & $\$ 13.08$ \\
\hline PF-condón & $\$ 27.10$ & $\$ 14.32$ & $\$ 27.10$ & $\$ 15.26$ & $\mathrm{np}$ & $\$ 15.26$ \\
\hline PF-depoprovera & $\$ 17.26$ & $\$ 17.70$ & $\$ 17.26$ & $\$ 19.56$ & $\mathrm{np}$ & $\$ 19.56$ \\
\hline PF-DIU & $\$ 6.54$ & $\$ 14.56$ & $\$ 6.54$ & $\$ 14.56$ & $\$ 10.72$ & $\$ 14.56$ \\
\hline PF-esterilización & np & np & np & np & $\$ 30.98$ & $\$ 30.78$ \\
\hline Sepsis & $\mathrm{np}$ & $\mathrm{np}$ & np & $\$ 59.86$ & $\$ 102.96$ & $\$ 104.54$ \\
\hline $\begin{array}{l}\text { Notas: } \\
\text { PMB: Paquete Madre Bebé } \\
\text { ITS: infecciones de transmisiór } \\
\text { PF: planificación familiar } \\
\text { DIU: dispositivo intrauterino } \\
\text { np: servicio no provisto } \\
\text { Costo en dólares estadounide }\end{array}$ & & & & & & \\
\hline
\end{tabular}

xico para la provisión de servicios de planificación familiar ${ }^{24}$ y atención del parto normal ${ }^{27}$ en la SSA, atención del parto en el Instituto Mexicano del Seguro Social ${ }^{25,26}$ y el costo de las servicios de diagnóstico y tratamiento de infecciones de transmisión sexual, servicios de salud reproductiva, atención prenatal del embarazo, atención del parto normal y del puerperio y atención del parto por cesárea y puerperio en el Seguro Popular de Salud. ${ }^{27}$

Por otro lado, al aplicarse el modelo PMB se recomienda una mayor inversión en contratación de personal médico, paramédico y administrativo, y una mayor compra de medicamentos para todos los establecimientos analizados, respecto al modelo actual. Si bien en este modelo gran proporción del presupuesto se destina a estos rubros, principalmente al de personal, el mayor gasto sugerido subraya la falta de insumos humanos y medicamentos para la provisión de servicios.

Adicionalmente, a pesar de ser una investigación piloto, con un tamaño de muestra reducido, sus resul- tados son útiles para mostrar la variabilidad de los costos entre diferentes niveles de atención en la SSA. Se observa así que el costo directo por caso es más alto mientras mayor capacidad tenga el establecimiento de salud, lo que justifica diseñar políticas de redistribución de pacientes como la propuesta por el PMB. Es de mencionarse que todas estas recomendaciones se mantienen aun cuando se varíen parámetros como el tiempo de trabajo del personal médico dedicado para cada intervención y el porcentaje del tiempo de trabajo del personal médico que se considera como tiempo muerto.

Se encontraron algunas dificultades metodológicas en la aplicación del Modelo de estimación de costos que pueden tener implicaciones en el costeo. Una primera dificultad se deriva de la falta de criterios claramente establecidos para la recopilación de información de los servicios provistos por los establecimientos de salud, como día-cama, por ejemplo. Otra dificultad se refiere a la inclusión de la productividad del personal 


\section{Costo total de los modelos actual y del Paquete Madre Bebé, por insumo en cada tipo de establecimiento, Jurisdicción Sanitaria III. Morelos, México, 2001}

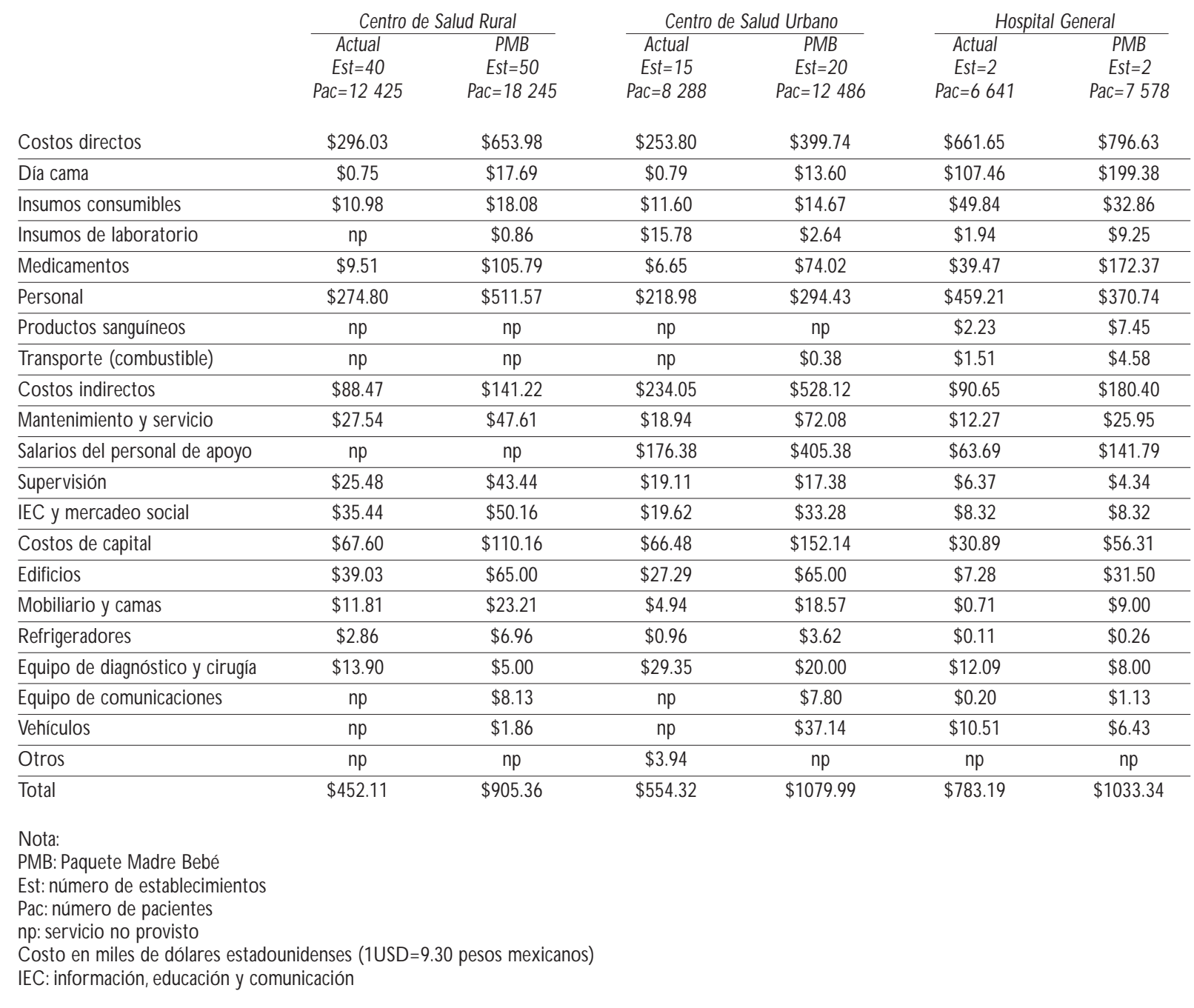

en los costos laborales, especialmente en casos en los que la capacidad del establecimiento puede ser excedida por la demanda. Si bien ambas limitaciones pueden generar alteraciones en los costos de cada modelo no alteran la relación de comparación entre ellos.

Además, es importante subrayar la necesidad de revisar supuestos propios del modelo PMB y hacerlos compatibles con la realidad y los objetivos de la SSA antes de implantarlo. Por ejemplo, en el modelo PMB se asume la existencia de un sistema de referencia funcional que, en el caso mexicano, no se encuentra completamente desarrollado. Considerar estos costos hubiese generado un incremento de la inversión en la atención materno infantil, debido a la relativa debilidad de la infraestructura de salud, la necesidad de invertir significativamente en esta área para que sea capaz de proveer atención apropiada (y de este modo, lograr que los centros de salud ofrezcan atención de partos), y la exigencia de incluir explícitamente estos costos en cualquier discusión de políticas.

Asimismo, se deben mencionar dificultades enfrentadas en la recolección de aquellos datos que también pudieran haber influido en la estimación de costos. En primer lugar, no se pudo obtener información sobre el manejo de complicaciones del aborto, pues su mención generó indisposición en el personal de salud entrevistado. En segundo lugar, ante la dificultad en el personal de salud para describir un caso basado en 
guías de tratamiento establecidas (caso promedio para el PMB), se capturó información para el tratamiento de un caso típico.

Las limitaciones antes mencionadas, aunadas a la poca homogeneidad de los establecimientos de salud en cada nivel de atención, condicionó la construcción de costos promedio por cada tipo de establecimiento. Sin embargo, a pesar de ellas, el uso del Modelo de estimación de costos mostró ser útil para obtener resultados que ilustren la relación entre el costo de la provisión actual de servicios de salud y la provisión bajo estándares internacionales.

Por lo anterior, es posible afirmar que el Modelo de estimación de costos es una herramienta práctica que puede emplearse para la toma de decisiones económicas que permitan determinar prioridades y, por tanto, la redistribución adecuada de recursos para la atención materno infantil entre diferentes tipos de establecimientos, por medio de la estandarización de las intervenciones que comprende el PMB. Sin embargo, su aplicación es limitada si su adaptación no responde a las características de cada sistema de atención.

Entre líneas futuras de investigación se encuentra el aplicar el Modelo de estimación de costos para costear intervenciones del Programa de Arranque Parejo en la Vida y el Seguro Popular de Salud, ampliando la muestra de este estudio. En particular, un número mayor de unidades no sólo brindaría una mayor representatividad a escala estatal, sino que si además se incluyen unidades de otras entidades federativas, también se podría analizar si los resultados obtenidos se pueden extrapolar a otras entidades. Asimismo, se podría considerar incluir un mayor número de tipos de establecimientos pues, por motivos de nuestro trabajo, se dejaron de lado centros de salud rural pequeños, y sólo se incluyeron los centros grandes en la cabecera municipal. De igual modo, una ampliación de este trabajo podría considerar comparar los costos de la atención materno infantil entre los distintos sistemas de provisión de la salud en el país.

Finalmente, queda pendiente analizar, junto con los resultados económicos de este estudio (primero en documentar la aplicación del Modelo de estimación de costos en México), temas sobre la implantación del PMB en el país, como por ejemplo el de los factores que influyen en la demanda de atención materno infantil y en la aceptación de los cambios propuestos por el PMB, un análisis de la calidad técnica de los estándares de atención que se generen (para hacerlos compatibles con el PMB) y un estudio de la efectividad del $\mathrm{PMB}$ en el contexto mexicano, que permita obtener resultados concluyentes para que los tomadores de de- cisiones tengan más elementos para decidir si se implanta o no el PMB.

\section{Agradecimientos}

Los autores desean agradecer al Hospital General de Cuautla, a los centros de salud en donde se efectuaron las entrevistas, a la Jurisdicción Sanitaria No. III y a los Servicios de Salud del Estado de Morelos, al facilitar el desarrollo de esta investigación. Asimismo desean agradecer a María de Lourdes Méndez por su invaluable participación en el levantamiento de los datos.

\section{Referencias}

1.W orld Health $\mathrm{O}$ rganization, D epartment of Reproductive Health and Research, Making pregnancy safer. G inebra:W HO. D isponible en: http:// www.who.int/reproductive-health/mpr/index.htm. [2003 agosto 5]. 2. Secretaría de Salud. La situación de la salud 2000. México, DF: SSA 2001.

3. Secretaría de Salud. Programa de salud reproductiva. México, DF: SSA; 1995.

4. Secretaría de Salud. Se fortalecen las acciones del Programa Arranque Parejo en laVida. Comunicado de Prensa N 0. 158. México, DF: SSA; 2003 julio 21. Disponible en: http://www.salud.gob.mx/ssa_app/noticias/ datos/2003-07-21_634.html. [2003 agosto 5].

5. Graham W J, Bell JS, Bullough CHW. Can skilled attendance at delivery reduce maternal mortality in developing countries? En: Brouwere V,Van Lerberghe W, ed. Safe motherhood strategies:A review of the evidence. Stud Health Serv O rgan Policy 2001;17:97-130.

6. Koblinsky MA, Campbell O, Heichelheim J. 0 rganizing delivery care: W hat works for safe motherhood? Bull W orld Health O rgan 1999;77(5):399-406.

7.W orld Health 0 rganization. Mother-Baby Package: Implementing safe motherhood in countries. Rev. 1. Ginebra:W HO , Division of Reproductive Health; 1994.

8. Microsoft Corporation. Microsoft Excel, versión 2000. Redmond (W A): Microsoft Corporation; 2000.

9. W orld Health 0 rganization. Mother-Baby Package costing spreadsheet.Versión 1.01. Ginebra:W HO ; 1999.

10.W orld Health 0 rganization. Mother-Baby Package costing spreadsheet user guide. Ginebra:W HO , D ivision of Reproductive Health; 1999.

11. Capra K, Lissner C, Seoane G, W eissman E, Fiedler J. Cost study for the Mother-Baby Package. La Paz, Bolivia: Mother Care Bolivia; 1998.

12. W eissman $E$, Sentumbwe-Mugisa 0 , M bonye AK, Lissner C. Costing safe motherhood in U ganda. En: Berer M, Sundari Ravindran TK, ed. Reproductive health matters: Safe motherhood initiatives: Critical issues. 0 xford: Blackwell Science; 1999:85-92.

13. Sosa S. Estimación de costos de la atención materno infantil en el primer nivel en una zona rural del estado de Morelos (tesis). México, DF: CIDE; 1999

14. Secretaría de Salud. Programa de acción: "Arranque Parejo en la Vida". México, DF:SSA; 2002.

15. Secretaría de Salud. Seguro Popular de Salud. México, DF: Seguro Popular de Salud. Disponible en: http://www.salud.gob.mx/apps/htdocs/ seguro_popular/catalogo_medico.htm. [2003 agosto 5]. 
16. A vila JL, Fuentes C, Tuirán R. Indices de marginación; 2000. México, DF:Conapo; 2001.

17. Post M. Preventing maternal mortality through emergency obstetric care. Support for analysis and research in Africa (SARA). Issues Paper; Silver Spring (MD): U nited States A gency for International D evelopment; 1997. Disponible en: http://www.dec.org/pdf_docs/PN ACB231.pdf. [2002 agosto 3].

18. Servicios de Salud de Morelos. Directorio de unidades de primer nivel de atención 2001. Cuautla, Morelos, México: Jurisdicción Sanitaria N o. III; 2001.

19. Secretaría de Salud. Programa de acción: "Salud Reproductiva". México, DF: SSA; 2002.

20. Secretaría de Salud. D eterminación de costos del Paquete Básico de Servicios de Salud (PBSS). México, DF: Dirección General de Extensión de Cobertura; 1998.

21. Banco de México. Indice de precios al consumidor. Por objeto del gasto y actividad económica. N acional. México, DF: Banco de México. Disponible en: http://www.banxico.gob.mx/elnfoFinanciera/ FSinfo Financiera.html. [2003 agosto 5].

22. Banco de México.Tipos de cambio. Información mensual.Tipo de cambio promedio del periodo. México, DF: Banco de México.
Disponible en: http://www.banxico.gob.mx/elnfoFinanciera/ FSinfo Financiera.html [2003 agosto 5].

23.The prevention of maternal mortality network. Situation analyses of emergency obstetric care: Examples from eleven operations research projects in W est A frica. Soc Sci Med 1995;40(5):657-667.

24. Hubacher D, Holtman M, Fuentes M, Pérez-Palacios G, Janowitz B. Increasing efficiency to meet future demand: Family planning services provided by the Mexican Ministry of Health. Int Fam Plann Perspect 1999;25(3):199-125

25. Arredondo A, D amián T, De Icaza E. Una aproximación al estudio de costos de servicios de salud en México. Salud Publica Mex 1995;37:437445

26. Instituto Mexicano del Seguro Social (s/f) Informe al Congreso de la Unión sobre los seguros y coberturas que administra el Instituto Mexicano del Seguro Social. 0 ctubre 2002. México, DF: Instituto Mexicano del Seguro Social. D isponible en: http://www.imss.gob.mx/ IMSS/Informes/Seguros_Coberturasç [2003 agosto 5].

27.Avila-Figueroa C, Gómez-Fraga S, Herrera BE, Sousa FA, Lozano AR. Estimación del costo de producción de servicios de prevención, diagnóstico y tratamiento médico. 2. México, D F: Secretaría de Salud; 2003. 